\title{
Corrigendum to "Evacuation after the Fukushima Daiichi Nuclear Power Plant Accident Is a Cause of Diabetes: Results from the Fukushima Health Management Survey"
}

\author{
Hiroaki Satoh, ${ }^{1,2}$ Tetsuya Ohira, ${ }^{2,3}$ Mitsuaki Hosoya,, ${ }^{2,4}$ Akira Sakai, ${ }^{2,5}$ \\ Tsuyoshi Watanabe, ${ }^{1,2}$ Akira Ohtsuru, ${ }^{2,6}$ Yukihiko Kawasaki, ${ }^{2,4}$ Hitoshi Suzuki, ${ }^{2,7}$ \\ Atsushi Takahashi, ${ }^{2,8}$ Gen Kobashi, ${ }^{9}$ Kotaro Ozasa, ${ }^{10}$ Seiji Yasumura, ${ }^{2,11}$ \\ Shunichi Yamashita, ${ }^{2,12}$ Kenji Kamiya, ${ }^{2,13}$ and Masafumi Abe $^{2}$ \\ ${ }^{1}$ Department of Nephrology, Hypertension, Diabetology, Endocrinology, and Metabolism, Fukushima Medical University, \\ Fukushima 960-1295, Japan \\ ${ }^{2}$ Radiation Medical Science Center for the Fukushima Health Management Survey, Fukushima Medical University, \\ Fukushima 960-1295, Japan \\ ${ }^{3}$ Department of Epidemiology, Fukushima Medical University, Fukushima 960-1295, Japan \\ ${ }^{4}$ Department of Pediatrics, Fukushima Medical University, Fukushima 960-1295, Japan \\ ${ }^{5}$ Department of Radiation Life Sciences, Fukushima Medical University, Fukushima 960-1295, Japan \\ ${ }^{6}$ Department of Radiation Health Management, Fukushima Medical University, Fukushima 960-1295, Japan \\ ${ }^{7}$ Department of Cardiology and Hematology, Fukushima Medical University, Fukushima 960-1295, Japan \\ ${ }^{8}$ Department of Gastroenterology and Rheumatology, Fukushima Medical University, Fukushima 960-1295, Japan \\ ${ }^{9}$ Department of Planning and Management, National Institute of Radiological Sciences, Fukushima Medical University, \\ Fukushima 960-1295, Japan \\ ${ }^{10}$ Department of Epidemiology, Radiation Effects Research Foundation, Fukushima Medical University, Fukushima 960-1295, Japan \\ ${ }^{11}$ Department of Public Health, Fukushima Medical University, Fukushima 960-1295, Japan \\ ${ }^{12}$ Atomic Bomb Disease Institute, Nagasaki University, Nagasaki, Japan \\ ${ }^{13}$ Research Institute for Radiation Biology and Medicine, Hiroshima University, Hiroshima, Japan \\ Correspondence should be addressed to Hiroaki Satoh; hiroakis-tky@umin.ac.jp
}

Received 1 July 2015; Accepted 30 July 2015

Copyright (c) 2015 Hiroaki Satoh et al. This is an open access article distributed under the Creative Commons Attribution License, which permits unrestricted use, distribution, and reproduction in any medium, provided the original work is properly cited.

In the paper titled "Evacuation after the Fukushima Daiichi Nuclear Power Plant Accident Is a Cause of Diabetes: Results from the Fukushima Health Management Survey" [1], the description of "multivariate logistic regression analysis" (Line 11) in the Abstract is incorrect. "Cox proportional hazards model" is the correct form mentioned in the description in Materials and Methods.

\section{References}

[1] H. Satoh, T. Ohira, M. Hosoya et al., "Evacuation after the fukushima daiichi nuclear power plant accident is a cause of diabetes: results from the fukushima health management survey," Journal of Diabetes Research, vol. 2015, Article ID 627390, 9 pages, 2015. 


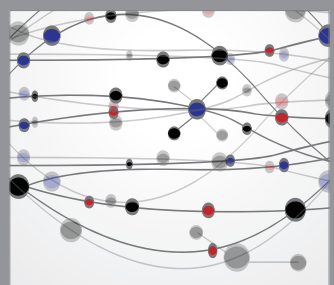

The Scientific World Journal
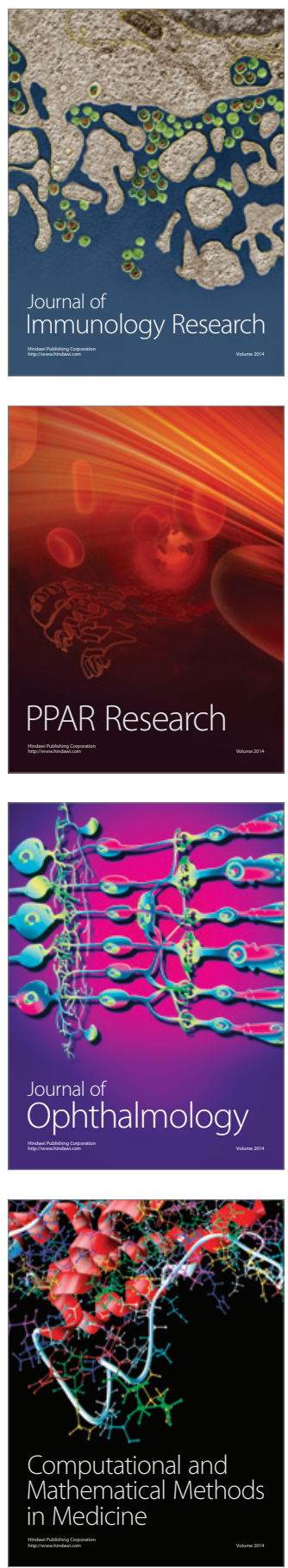

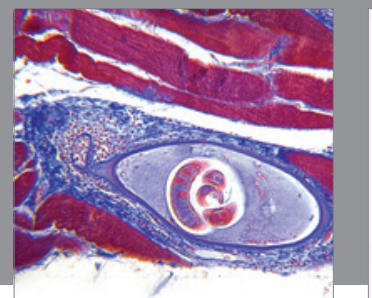

Gastroenterology

Research and Practice
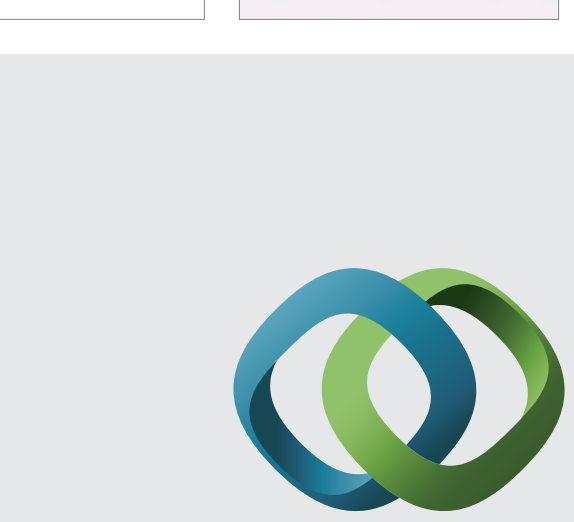

\section{Hindawi}

Submit your manuscripts at

http://www.hindawi.com
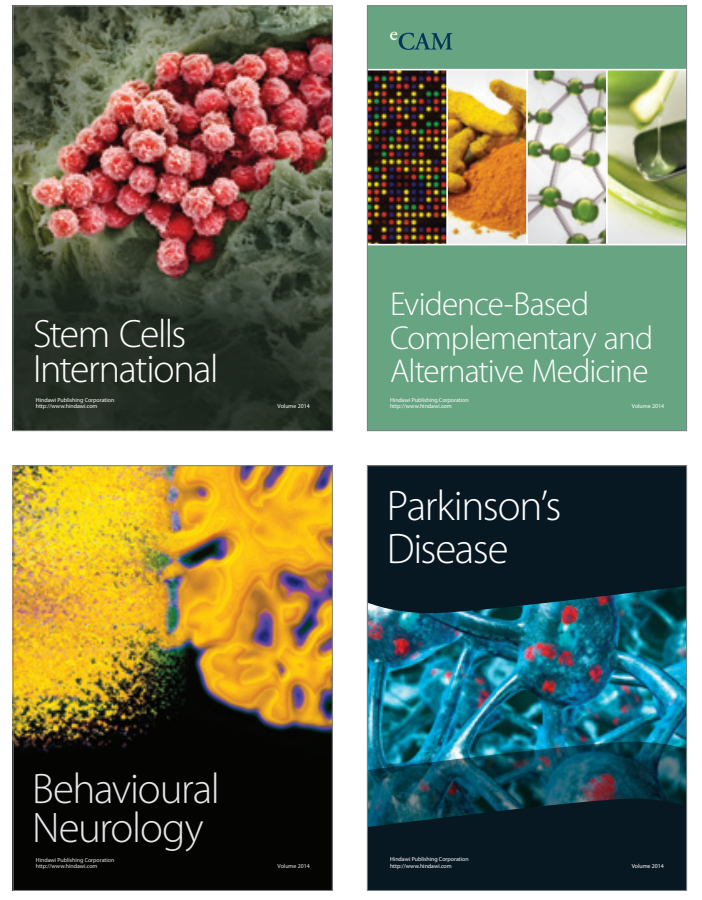
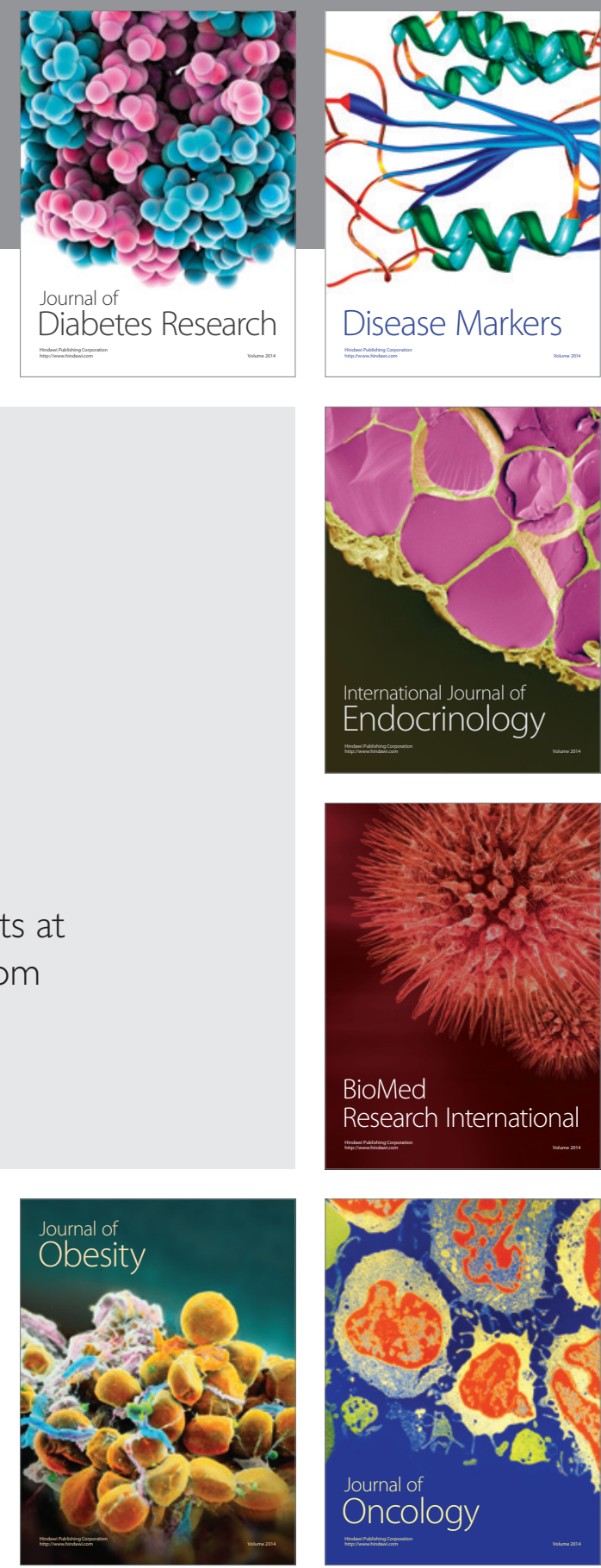

Disease Markers
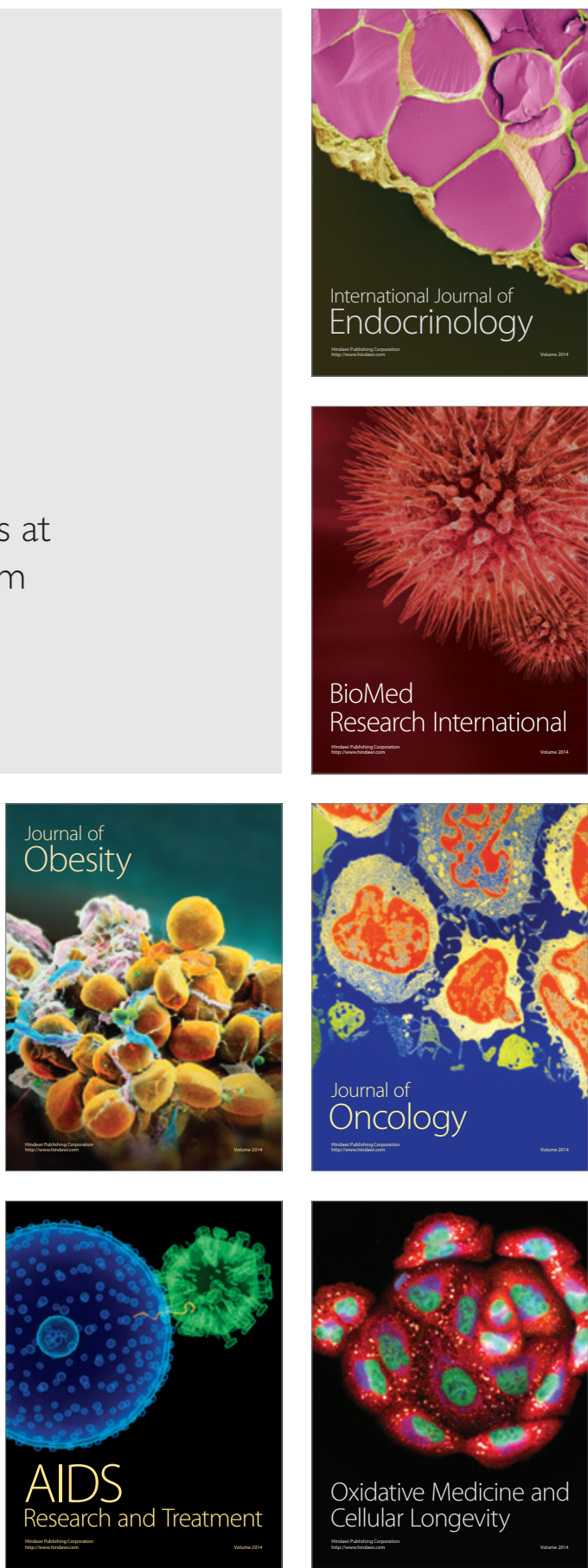